\title{
WEIGHTED DIVISOR SUMS AND BESSEL FUNCTION SERIES
}

\author{
BRUCE C. BERNDT ${ }^{1}$ and ALEXANDRU ZAHARESCU
}

\begin{abstract}
On page 335 in his lost notebook, Ramanujan records without proof an identity involving a finite trigonometric sum and a doubly infinite series of ordinary Bessel functions. We provide the first published proof of this result. The identity yields as corollaries representations of weighted divisor sums, in particular, the summatory function for $r_{2}(n)$, the number of representations of the positive integer $n$ as a sum of two squares.
\end{abstract}

\section{INTRODUCTION}

In this paper we establish identities that express certain weighted divisor sums as double series of Bessel functions. Our main result, stated in Theorem 1.1 below, is an identity claimed by Ramanujan on page 335 in his lost notebook [14], for which no indication of a proof is given, and which has not been heretofore proved. (Technically, page 335 is not in Ramanujan's lost notebook; this page is a fragment published by Narosa with the original lost notebook.) The identity involves the ordinary Bessel function $J_{1}(z)$, where

$$
J_{\nu}(z):=\sum_{n=0}^{\infty} \frac{(-1)^{n}}{n ! \Gamma(\nu+n+1)}\left(\frac{z}{2}\right)^{\nu+2 n}, \quad 0<|z|<\infty, \quad \nu \in \mathbb{C} .
$$

To state Ramanujan's claim, we need to first define

$$
F(x)= \begin{cases}{[x],} & \text { if } x \text { is not an integer } \\ x-\frac{1}{2}, & \text { if } x \text { is an integer }\end{cases}
$$

where, as customary, $[x]$ is the greatest integer less than or equal to $x$.

Theorem 1.1. If $0<\theta<1$ and $x>0$, then

$$
\begin{aligned}
\sum_{n=1}^{\infty} F\left(\frac{x}{n}\right) \sin (2 \pi n \theta) & =\pi x\left(\frac{1}{2}-\theta\right)-\frac{1}{4} \cot (\pi \theta) \\
& +\frac{1}{2} \sqrt{x} \sum_{n=0}^{\infty} \sum_{m=1}^{\infty}\left\{\frac{J_{1}(4 \pi \sqrt{m(n+\theta) x})}{\sqrt{m(n+\theta)}}-\frac{J_{1}(4 \pi \sqrt{m(n+1-\theta) x})}{\sqrt{m(n+1-\theta)}}\right\} .
\end{aligned}
$$

\footnotetext{
${ }^{1}$ Research partially supported by grant MDA H92830-04-1-0027 from the National Security Agency.
} 
We emphasize that (1.3) is not the formulation given by Ramanujan in his lost notebook. Ramanujan claims that

$$
\begin{aligned}
& {\left[\frac{x}{1}\right] \sin (2 \pi \theta)+\left[\frac{x}{2}\right] \sin (4 \pi \theta)+\left[\frac{x}{3}\right] \sin (6 \pi \theta)+\left[\frac{x}{4}\right] \sin (8 \pi \theta)+\cdots} \\
& =\pi x\left(\frac{1}{2}-\theta\right)-\frac{1}{4} \cot (\pi \theta)+\frac{1}{2} \sqrt{x} \sum_{m=1}^{\infty}\left\{\frac{J_{1}(4 \pi \sqrt{m \theta x})}{\sqrt{m \theta}}-\frac{J_{1}(4 \pi \sqrt{m(1-\theta) x})}{\sqrt{m(1-\theta)}}+\right. \\
& \left.+\frac{J_{1}(4 \pi \sqrt{m(1+\theta) x})}{\sqrt{m(1+\theta)}}-\frac{J_{1}(4 \pi \sqrt{m(2-\theta) x})}{\sqrt{m(2-\theta)}}+\frac{J_{1}(4 \pi \sqrt{m(2+\theta) x})}{\sqrt{m(2+\theta)}}-\cdots\right\}
\end{aligned}
$$

"where $[x]$ denotes the greatest integer in $x$ if $x$ is not an integer and $x-\frac{1}{2}$ if $x$ is an integer." Since Ramanujan employed the notation $[x]$ in a nonstandard fashion, we have introduced the notation (1.2). Also, note that the order of summation in the double series on the right side of (1.3) has been reversed from that given by Ramanujan on the right side of (1.4). It could be that in an unorthodox fashion, Ramanujan meant that the distributive law must be employed in (1.4), and so his view of (1.4) may actually be the same as ours in (1.3). Extensive numerical calculations confirmed, in fact, that if Ramanujan's identity were to be true, our version (1.3) must be correct. Moreover, calculations indicate that Ramanujan's double series, under the most natural interpretation of (1.4), converges to another value, possibly 0; thus, the two orders of summation apparently yield different results.

Note that the series on the left-hand side of (1.3) is finite and discontinuous if $x$ is an integer. To examine the right-hand side, we recall that $[17$, p. 199], as $x \rightarrow \infty$,

$$
J_{\nu}(x) \sim\left(\frac{2}{\pi x}\right)^{1 / 2} \cos \left(x-\frac{1}{2} \nu \pi-\frac{1}{4} \pi\right) .
$$

Hence, as $m, n \rightarrow \infty$, the terms of the double series on the right-hand side of (1.3) are asymptotically equal to

$$
\frac{1}{\pi \sqrt{2} x^{1 / 4} m^{3 / 4}}\left(\frac{\cos \left(4 \pi \sqrt{m(n+\theta) x}-\frac{3}{4} \pi\right)}{(n+\theta)^{3 / 4}}-\frac{\cos \left(4 \pi \sqrt{m(n+1-\theta) x}-\frac{3}{4} \pi\right)}{(n+1-\theta)^{3 / 4}}\right) .
$$

Thus, if indeed the double series on the right side of (1.3) does converge, it converges conditionally and not absolutely.

Before proving (1.3), it is natural to ask what led Ramanujan to the double series on the right side of (1.3).

Let $r_{2}(n)$ denote the number of representations of the positive integer $n$ as a sum of two squares. In connection with his significant work on the famous circle problem, in 1915, Hardy [9], [10, pp. 243-263] proved that

$$
\sum_{0 \leq n \leq x}{ }^{\prime} r_{2}(n)=\pi x+\sum_{n=1}^{\infty} r_{2}(n)\left(\frac{x}{n}\right)^{1 / 2} J_{1}(2 \pi \sqrt{n x}),
$$

where the prime $/$ on the summation sign on the left side indicates that if $x$ is an integer, only $\frac{1}{2} r_{2}(x)$ is counted. Observe that the series on the right side of (1.6) is 
similar to the inner series on the right side of (1.3). Moreover, the sums on the left side in each formula are finite sums over $n \leq x$. Ramanujan might therefore have derived (1.3) in anticipation of applying it to the circle problem. When Hardy published his paper [9] in 1915, Ramanujan was at Cambridge University, and he must have been intrigued by Hardy's identity. In this same paper, Hardy relates a beautiful identity of Ramanujan connected with $r_{2}(n)$, namely, for $a, b>0,[9$, p. 283], [10, p. 263],

$$
\sum_{n=0}^{\infty} \frac{r_{2}(n)}{\sqrt{n+a}} e^{-2 \pi \sqrt{(n+a) b}}=\sum_{n=0}^{\infty} \frac{r_{2}(n)}{\sqrt{n+b}} e^{-2 \pi \sqrt{(n+b) a}}
$$

which is not given elsewhere in any of Ramanujan's published or unpublished work.

To see the connection between (1.3) and divisor sums, note that if the factor $\sin (2 \pi n \theta)$ were missing on the left side, then the sum on the left side of (1.3) would coincide with the number of integer points $(n, l)$ with $n, l \geq 1$ and $n l \leq x$, where the pairs $(n, l)$ satisfying $n l=x$ are counted with weight $1 / 2$. It follows that

$$
\sum_{n=1}^{\infty} F\left(\frac{x}{n}\right)=\sum_{1 \leq n \leq x}^{\prime} d(n),
$$

where $d(n)$ denotes the number of divisors of $n$, and the prime $/$ on the summation sign indicates that if $x$ is an integer, only $\frac{1}{2} d(x)$ is counted.

Therefore one may interpret the left side of (1.3) as a weighted divisor sum. In this way we can also obtain, for instance, exact formulas for divisor sums twisted by Dirichlet characters. If $q$ is a positive integer and $\chi$ is an odd primitive character modulo $q$, and if we denote

$$
d_{\chi}(n)=\sum_{k \mid n} \chi(k)
$$

then we obtain a formula for the corresponding twisted divisor sum $\sum_{1 \leq n \leq x}{ }^{\prime} d_{\chi}(n)$, where the prime $/$ on the summation sign has the same meaning as before.

Theorem 1.2. Let $q$ be a positive integer, let $\chi$ be an odd primitive character modulo $q$, and let $d_{\chi}(n)$ be defined by (1.7). Then, for any $x>0$,

$$
\begin{aligned}
& \sum_{1 \leq n \leq x}{ }^{\prime} d_{\chi}(n)=L(1, \chi) x-\frac{i \tau(\chi)}{2 \pi} L(1, \bar{\chi}) \\
& +\frac{i \sqrt{x}}{\tau(\bar{\chi})} \sum_{1 \leq h<q / 2} \bar{\chi}(h) \sum_{n=0}^{\infty} \sum_{m=1}^{\infty}\left\{\frac{J_{1}\left(4 \pi \sqrt{m\left(n+\frac{h}{q}\right) x}\right)}{\sqrt{m\left(n+\frac{h}{q}\right)}}-\frac{J_{1}\left(4 \pi \sqrt{m\left(n+1-\frac{h}{q}\right) x}\right)}{\sqrt{m\left(n+1-\frac{h}{q}\right)}}\right\},
\end{aligned}
$$

where $L(s, \chi)$ denotes the Dirichlet L-function associated with the character $\chi$, and $\tau(\bar{\chi})$ denotes the Gauss sum

$$
\tau(\bar{\chi})=\sum_{m=1}^{q} \bar{\chi}(m) e^{2 \pi i m / q}
$$


In particular, let $\chi$ be the nonprincipal Dirichlet character modulo 4. Recall Jacobi's famous formula [12],

$$
r_{2}(n)=4 \sum_{\substack{d \mid n \\ d \text { odd }}}(-1)^{(d-1) / 2}=4 d_{\chi}(n)
$$

for all positive integers $n$. Then by Theorem 1.2 we can conclude the following representation for $\sum_{0 \leq n \leq x}{ }^{\prime} r_{2}(n)$.

Corollary 1.3. For any $x>0$,

$$
\sum_{0 \leq n \leq x}^{\prime} r_{2}(n)=\pi x+2 \sqrt{x} \sum_{n=0}^{\infty} \sum_{m=1}^{\infty}\left\{\frac{J_{1}\left(4 \pi \sqrt{m\left(n+\frac{1}{4}\right) x}\right)}{\sqrt{m\left(n+\frac{1}{4}\right)}}-\frac{J_{1}\left(4 \pi \sqrt{m\left(n+\frac{3}{4}\right) x}\right)}{\sqrt{m\left(n+\frac{3}{4}\right)}}\right\} .
$$

Recall that the famous circle problem is to determine the precise order of magnitude for the "error term" $P(x)$ defined by

$$
\sum_{0 \leq n \leq x}{ }^{\prime} r_{2}(n)=\pi x+P(x)
$$

In [9], Hardy showed that $P(x) \neq O\left((x \log x)^{1 / 4}\right)$, as $x$ tends to $\infty$. Most efforts toward obtaining an upper bound for $P(x)$ have ultimately rested upon (1.6), (1.5), and methods of estimating the resulting trigonometric series. At present, the best result in this direction has been established by N. M. Huxley [11], who proved that $P(x)=O\left(x^{131 / 408}\right)$. Will (1.10) lead to greater success in estimating $P(x)$ than (1.6) has been? A possible advantage in using (1.10) is that $r_{2}(n)$ does not occur on the right side of (1.10), as in (1.6). On the other hand, double series are likely to be more difficult to estimate than a single infinite series. Analogues of the problem of estimating the error term $P(x)$ for $\sum_{0 \leq n \leq x}{ }^{\prime} r_{2}(n)$ exist for many other arithmetical functions $a(n)$ generated by Dirichlet series satisfying a functional equation involving the gamma function $\Gamma(s)$. See, for example, a paper by $K$. Chandrasekharan and R. Narasimhan [7]. Again, representations for the summatory function $\sum_{n \leq x} a(n)$ in terms of Bessel functions play a critical role. However, in many cases $\sum_{n \leq x} a(n)$ may not be representable in terms of an infinite series of Bessel functions, but, for sufficiently large positive numbers $q, \sum_{n \leq x} a(n)(x-n)^{q}$ can be so represented. See, for example, [6], [1], and [2]. Is there an analogue of (1.8) for $\sum_{1 \leq n \leq x}{ }^{\prime} d_{\chi}(n)(x-n)^{q}$ for complex $q$ ? If $a(n)$ is generated by a Dirichlet series satisfying a functional equation involving $\Gamma(s)$, is there an analogue of (1.8) for $\sum_{n \leq x} a(n)$; if so, can it be extended to provide a representation for $\sum_{n \leq x} a(n)(x-n)^{q}$, for complex $q$, as a double series of Bessel functions? Bessel function identities for $\sum_{n \leq x} a(n)(x-n)^{q}$ are, in fact, equivalent to the corresponding Dirichlet series satisfying a functional equation involving $\Gamma(s)[6]$. 


\section{Proofs of Theorem 1.2 And Corollary 1.3}

We derive Theorem 1.2 from Theorem 1.1. First, we rewrite the given twisted divisor sum in terms of the function $F$, namely,

$$
\sum_{1 \leq n \leq x}{ }^{\prime} d_{\chi}(n)=\sum_{n=1}^{\infty} F\left(\frac{x}{n}\right) \chi(n) .
$$

Secondly, we write $\chi(n)$ as a linear combination of values of $\sin (2 \pi n \theta)$ for certain appropriate values of $\theta$. Thus, using the well known identity [5, p. 9, Thm. 1.1.3]

$$
\chi(n)=\frac{1}{\tau(\bar{\chi})} \sum_{h=1}^{q} \bar{\chi}(h) e^{2 \pi i n h / q}
$$

where

$$
\tau(\chi)=\sum_{m=1}^{q} \chi(m) e^{2 \pi i m / q}
$$

and the fact that $\chi$ is odd, we see that

$$
\chi(n)=\frac{2 i}{\tau(\bar{\chi})} \sum_{1 \leq h<q / 2} \bar{\chi}(h) \sin \left(\frac{2 \pi n h}{q}\right) .
$$

Third, recall the classical formulas (e. g., see [4, Thms. 3.2 and 13.3])

$$
\sum_{1 \leq h<q / 2} \bar{\chi}(h)=\frac{i \tau(\bar{\chi})}{\pi}(\chi(2)-2) L(1, \chi)
$$

and

$$
\sum_{1 \leq h<q / 2} \bar{\chi}(h) h=\frac{i \tau(\bar{\chi}) q}{2 \pi}(\chi(2)-1) L(1, \chi) .
$$

Combining the last two equalities, we find that

$$
L(1, \chi)=\frac{2 \pi i}{\tau(\bar{\chi})} \sum_{1 \leq h<q / 2} \bar{\chi}(h)\left(\frac{1}{2}-\frac{h}{q}\right) .
$$

Lastly, recall the elementary identity

$$
\cot \left(\frac{\pi h}{q}\right)=-\frac{2}{q} \sum_{j=1}^{q-1} j \sin \left(\frac{2 \pi j h}{q}\right) .
$$

Multiplying both sides of (2.6) by $\bar{\chi}(h)$, summing on $h, 1 \leq h<q / 2$, inverting the order of summation, using (2.2), recalling that $\chi$ is odd, employing the identities (2.3) and (2.4), and appealing to the fact that $\tau(\chi) \tau(\bar{\chi})=-q$ for odd $\chi[5$, Thm. 1.4(a), p. 10], we find that

$$
\sum_{1 \leq h<q / 2} \bar{\chi}(h) \cot \left(\frac{\pi h}{q}\right)=-\frac{2}{q} \sum_{j=1}^{q-1} j \sum_{1 \leq h<q / 2} \bar{\chi}(h) \sin \left(\frac{2 \pi j h}{q}\right)
$$




$$
\begin{aligned}
& =-\frac{\tau(\bar{\chi})}{i q} \sum_{j=1}^{q-1} j \chi(j) \\
& =-\frac{\tau(\bar{\chi})}{i q}\left(\sum_{0<j<q / 2}+\sum_{q / 2<j<q}\right) j \chi(j) \\
& =-\frac{\tau(\bar{\chi})}{i q}\left(2 \sum_{0<j<q / 2} j \chi(j)-q \sum_{0<j<q / 2} \chi(j)\right) \\
& =-\frac{\tau(\bar{\chi})}{i q}\left(\frac{i q \tau(\chi)}{\pi}(\bar{\chi}(2)-1) L(1, \bar{\chi})-\frac{i q \tau(\chi)}{\pi}(\bar{\chi}(2)-2) L(1, \bar{\chi})\right) \\
& =\frac{q}{\pi} L(1, \bar{\chi}) .
\end{aligned}
$$

Hence, from (2.7),

$$
-\frac{i}{2 \tau(\bar{\chi})} \sum_{1 \leq h<q / 2} \bar{\chi}(h) \cot \left(\frac{\pi h}{q}\right)=-\frac{i q}{2 \pi \tau(\bar{\chi})} L(1, \bar{\chi})=\frac{i \tau(\chi)}{2 \pi} L(1, \bar{\chi}) .
$$

Finally, let $\theta=h / q$, multiply both sides of $(1.3)$ by $2 i \bar{\chi}(h) / \tau(\bar{\chi})$, and sum on $h, 1 \leq$ $h<q / 2$. On the new left side of (1.3), we find that

$$
\frac{2 i}{\tau(\bar{\chi})} \sum_{1 \leq h<q / 2} \bar{\chi}(h) \sum_{n=1}^{\infty} \chi(n) F\left(\frac{x}{n}\right) \sin \left(\frac{2 \pi n h}{q}\right)=\sum_{n=1}^{\infty} F\left(\frac{x}{n}\right)=\sum_{1 \leq n \leq x}{ }^{\prime} d_{\chi}(n),
$$

by (2.2) and (2.1). On the new right side of (1.3), we use (2.5) and (2.8) to deduce the right side of (1.8). This then completes the proof of Theorem 1.2.

To derive Corollary 1.3, first note that if $\chi=\chi_{4}$, the nonprincipal primitive character of modulus 4 , then $\tau\left(\chi_{4}\right)=2 i$. Next, multiply (1.8) by 4 , use (1.9), and add 1 to both sides to complete the proof.

\section{Reduction to tRigonometric FunCtions}

The remainder of this paper is devoted to a proof of Theorem 1.1.

In this section we first convert (1.3) into an identity for a double series of trigonometric functions, Theorem 3.1, which is perhaps as intriguing as (1.3) and in which Bessel functions do not appear. Then we proceed to show that this double series converges uniformly for $0 \leq \theta \leq 1$. The proof of convergence is considerably involved and will be postponed to the next section. In Section 4 we then use the fact that if a series converges uniformly, its Fourier series coefficients uniquely determine the sum of the series. Thus, in the last part of our proof, we establish the Fourier series on the left side of (1.3).

Next, we convert (1.3) into an identity for a double series of trigonometric functions. To proceed, we employ Poisson's summation formula [16, pp. 60-61]. If $f(t)$ is 
continuous and of bounded variation on $[0, \infty)$, and if $\int_{0}^{\infty} f(t) d t$ exists, then

$$
\frac{1}{2} f(0)+\sum_{m=1}^{\infty} f(m)=\int_{0}^{\infty} f(t) d t+2 \sum_{m=1}^{\infty} \int_{0}^{\infty} f(t) \cos (2 \pi m t) d t
$$

Let us apply (3.1) with

$$
f(t):=\frac{J_{1}(4 \pi \sqrt{t(n+\theta) x})}{\sqrt{t(n+\theta)}} .
$$

Making the change of variable $u=4 \pi \sqrt{t(n+\theta) x}$ and using the differentiation formula $J_{1}(u)=-J_{0}^{\prime}(u)$, which is easily derived from (1.1), we find that

$$
\begin{aligned}
\int_{0}^{\infty} f(t) d t & =\frac{1}{2 \pi(n+\theta) \sqrt{x}} \int_{0}^{\infty} J_{1}(u) d u \\
& =-\frac{1}{2 \pi(n+\theta) \sqrt{x}} \int_{0}^{\infty} J_{0}^{\prime}(u) d u=\frac{1}{2 \pi(n+\theta) \sqrt{x}}
\end{aligned}
$$

where the evaluation of the integral on the far right side is a consequence of (1.5) and (1.1). To evaluate the integral

$$
\int_{0}^{\infty} f(t) \cos (2 \pi m t) d t=\int_{0}^{\infty} \frac{J_{1}(4 \pi \sqrt{t(n+\theta) x})}{\sqrt{t(n+\theta)}} \cos (2 \pi m t) d t
$$

we appeal to the formula [8, p. 771 , formula 6.725 , no. 2$]$

$$
\int_{0}^{\infty} \frac{J_{\nu}(a \sqrt{t})}{\sqrt{t}} \cos (b t) d t=\sqrt{\frac{\pi}{b}} \cos \left(\frac{a^{2}}{8 b}-\frac{\nu \pi}{4}-\frac{\pi}{4}\right) J_{\nu / 2}\left(\frac{a^{2}}{8 b}\right),
$$

where Re $\nu>-1$ and $a, b>0$. Hence, employing (3.5) with $a=4 \pi \sqrt{(n+\theta) x}$, $b=2 \pi m$, and $\nu=1$ and then using the familiar formula

$$
J_{1 / 2}(x)=\sqrt{\frac{2}{\pi x}} \sin x
$$

which is easily derivable from the definition (1.1), we find from (3.5) that

$$
\begin{aligned}
\int_{0}^{\infty} f(t) \cos (2 \pi m t) d t & =\sqrt{\frac{1}{2 m(n+\theta)}} \cos \left(\frac{\pi(n+\theta) x}{m}-\frac{\pi}{2}\right) J_{1 / 2}\left(\frac{\pi(n+\theta) x}{m}\right) \\
& =\frac{1}{\pi(n+\theta) \sqrt{x}} \sin ^{2}\left(\frac{\pi(n+\theta) x}{m}\right) .
\end{aligned}
$$

Lastly, from the definition of $f(t)$,

$$
f(0)=\lim _{t \rightarrow 0} \frac{2 \pi \sqrt{t(n+\theta) x}}{\sqrt{t(n+\theta)}}=2 \pi \sqrt{x} .
$$


Hence, using (3.7), (3.3), and (3.6) in (3.1), we find that

$$
\begin{aligned}
& \pi \sqrt{x}+\sum_{m=1}^{\infty} \frac{J_{1}(4 \pi \sqrt{m(n+\theta) x})}{\sqrt{m(n+\theta)}} \\
& \quad=\frac{1}{2 \pi(n+\theta) \sqrt{x}}+\frac{2}{\pi(n+\theta) \sqrt{x}} \sum_{m=1}^{\infty} \sin ^{2}\left(\frac{\pi(n+\theta) x}{m}\right) .
\end{aligned}
$$

Similarly,

$$
\begin{aligned}
\pi \sqrt{x}+\sum_{m=1}^{\infty} & \frac{J_{1}(4 \pi \sqrt{m(n+1-\theta) x})}{\sqrt{m(n+1-\theta)}} \\
& =\frac{1}{2 \pi(n+1-\theta) \sqrt{x}}+\frac{2}{\pi(n+1-\theta) \sqrt{x}} \sum_{m=1}^{\infty} \sin ^{2}\left(\frac{\pi(n+1-\theta) x}{m}\right) .
\end{aligned}
$$

Subtracting (3.9) from (3.8), we deduce that

$$
\begin{aligned}
& \sum_{m=1}^{\infty}\left\{\frac{J_{1}(4 \pi \sqrt{m(n+\theta) x})}{\sqrt{m(n+\theta)}}-\frac{J_{1}(4 \pi \sqrt{m(n+1-\theta) x})}{\sqrt{m(n+1-\theta)}}\right\} \\
= & \frac{1}{2 \pi \sqrt{x}}\left(\frac{1}{n+\theta}-\frac{1}{n+1-\theta}\right) \\
& +\frac{2}{\pi \sqrt{x}} \sum_{m=1}^{\infty}\left(\frac{1}{n+\theta} \sin ^{2}\left(\frac{\pi(n+\theta) x}{m}\right)-\frac{1}{n+1-\theta} \sin ^{2}\left(\frac{\pi(n+1-\theta) x}{m}\right)\right) .
\end{aligned}
$$

We now sum both sides of (3.10) on $n, 0 \leq n<\infty$. Now,

$$
\sum_{n=0}^{\infty}\left(\frac{1}{n+\theta}-\frac{1}{n+1-\theta}\right)=\lim _{N \rightarrow \infty} \sum_{n=-N}^{N} \frac{1}{n+\theta}=\pi \cot (\pi \theta) .
$$

Hence, so far we have shown that

$$
\begin{aligned}
& S(\theta, x):=\sum_{n=0}^{\infty} \sum_{m=1}^{\infty}\left\{\frac{J_{1}(4 \pi \sqrt{m(n+\theta) x})}{\sqrt{m(n+\theta)}}-\frac{J_{1}(4 \pi \sqrt{m(n+1-\theta) x})}{\sqrt{m(n+1-\theta)}}\right\} \\
= & \frac{1}{2 \sqrt{x}} \cot (\pi \theta) \\
& +\frac{2}{\pi \sqrt{x}} \sum_{n=0}^{\infty} \sum_{m=1}^{\infty}\left(\frac{1}{n+\theta} \sin ^{2}\left(\frac{\pi(n+\theta) x}{m}\right)-\frac{1}{n+1-\theta} \sin ^{2}\left(\frac{\pi(n+1-\theta) x}{m}\right)\right) .
\end{aligned}
$$


Multiplying both sides of (3.11) by $\frac{1}{2} \sqrt{x}$, we write (3.11) in the equivalent form

$$
\begin{aligned}
& \frac{1}{2} \sqrt{x} S(\theta, x)=\frac{1}{4} \cot (\pi \theta) \\
& +\frac{1}{\pi} \sum_{n=0}^{\infty} \sum_{m=1}^{\infty}\left(\frac{1}{n+\theta} \sin ^{2}\left(\frac{\pi(n+\theta) x}{m}\right)-\frac{1}{n+1-\theta} \sin ^{2}\left(\frac{\pi(n+1-\theta) x}{m}\right)\right) .
\end{aligned}
$$

Comparing (3.12) with (1.3), we see that it suffices to prove the following theorem.

Theorem 3.1. For $0<\theta<1$ and $x>0$,

$$
\begin{aligned}
& \sum_{n=1}^{\infty} F\left(\frac{x}{n}\right) \sin (2 \pi n \theta)-\pi x\left(\frac{1}{2}-\theta\right) \\
& =\frac{1}{\pi} \sum_{n=0}^{\infty} \sum_{m=1}^{\infty}\left(\frac{1}{n+\theta} \sin ^{2}\left(\frac{\pi(n+\theta) x}{m}\right)-\frac{1}{n+1-\theta} \sin ^{2}\left(\frac{\pi(n+1-\theta) x}{m}\right)\right) .
\end{aligned}
$$

The identity (3.8) was, in fact, first proved by S. L. Segal [15]. The proof of (3.8) that we have given is due to Berndt [3, pp. 158-162], who actually proved more general identities using the character Poisson summation formula. See also a related paper by T. Kano [13].

In the following paragraph, we outline our methods in proving Theorem 3.1.

Fix $x>0$ and allow $\theta$ to vary in $(0,1)$. It will be convenient to set $\theta=\frac{1}{2}+t,-\frac{1}{2}<$ $t<\frac{1}{2}$. Then (3.13) takes the shape

$$
\begin{array}{r}
\frac{1}{\pi} \sum_{n=0}^{\infty} \sum_{m=1}^{\infty}\left(\frac{1}{n+\frac{1}{2}+t} \sin ^{2}\left(\frac{\pi x\left(n+\frac{1}{2}+t\right)}{m}\right)\right. \\
\left.=\frac{1}{n+\frac{1}{2}-t} \sin ^{2}\left(\frac{\pi x\left(n+\frac{1}{2}-t\right)}{m}\right)\right) \\
=\sum_{n=1}^{\infty}(-1)^{n} F\left(\frac{x}{n}\right) \sin (2 \pi n t)+\pi x t
\end{array}
$$

It is easily seen that the inner sum on the left side of (3.14) converges for any fixed $n$. Set

$$
f_{n}(t):=\sum_{m=1}^{\infty}\left(\frac{1}{n+\frac{1}{2}+t} \sin ^{2}\left(\frac{\pi x\left(n+\frac{1}{2}+t\right)}{m}\right)-\frac{1}{n+\frac{1}{2}-t} \sin ^{2}\left(\frac{\pi x\left(n+\frac{1}{2}-t\right)}{m}\right)\right) .
$$

Each function $f_{n}(t), n \geq 1$, is continuous on the compact interval $\left[-\frac{1}{2}, \frac{1}{2}\right]$. Also, $f_{0}(t)$ is well defined and continuous on $\left(-\frac{1}{2}, \frac{1}{2}\right)$ and can be extended by continuity to $\left[-\frac{1}{2}, \frac{1}{2}\right]$. If we can show that $\sum_{n=0}^{\infty} f_{n}(t)$ converges uniformly on $\left[-\frac{1}{2}, \frac{1}{2}\right]$, then

$$
f(t):=\sum_{n=0}^{\infty} f_{n}(t), \quad t \in\left[-\frac{1}{2}, \frac{1}{2}\right]
$$

will be continuous on $\left[-\frac{1}{2}, \frac{1}{2}\right]$. Then, by $(3.14)$, we need to prove that

$$
\frac{1}{\pi} f(t)=\sum_{n=1}^{\infty}(-1)^{n} F\left(\frac{x}{n}\right) \sin (2 \pi n t)+\pi x t .
$$


Since both sides of (3.17) are continuous functions of $t$, and since the Fourier coefficients of a continuous function uniquely determine the function, (3.17) will be established if we can show that both sides of (3.17) have the same Fourier coefficients. Moreover, if we can show that the sum on the left side of (3.17) is uniformly convergent on $\left[-\frac{1}{2}, \frac{1}{2}\right]$, then

$$
\int_{-1 / 2}^{1 / 2} f(t) e^{2 \pi i k t} d t=\sum_{n=0}^{\infty} \int_{-1 / 2}^{1 / 2} f_{n}(t) e^{2 \pi i k t} d t
$$

\section{The CONVERGENCE PROBLEM}

We now address the task of showing the uniform convergence of the series in (3.16). For all (large) $n$, write

$$
f_{n}(t)=A_{n}(t)+B_{n}(t)+C_{n}(t)+D_{n}(t), \quad-\frac{1}{2} \leq t \leq \frac{1}{2},
$$

where

$$
\begin{aligned}
A_{n}(t) & :=\sum_{1 \leq m \leq n / \log ^{5} n} c_{m, n}(t), \\
B_{n}(t) & :=\sum_{n / \log ^{5} n<m \leq n \log ^{2} n} c_{m, n}(t), \\
C_{n}(t) & :=\sum_{n \log ^{2} n<m<n^{3 / 2}} c_{m, n}(t), \\
D_{n}(t) & :=\sum_{n^{3 / 2} \leq m} c_{m, n}(t),
\end{aligned}
$$

where

$$
c_{m, n}(t):=\frac{1}{n+\frac{1}{2}+t} \sin ^{2}\left(\frac{\pi x\left(n+\frac{1}{2}+t\right)}{m}\right)-\frac{1}{n+\frac{1}{2}-t} \sin ^{2}\left(\frac{\pi x\left(n+\frac{1}{2}-t\right)}{m}\right) .
$$

We now show that each of the series $A_{n}(t), B_{n}(t), C_{n}(t)$, and $D_{n}(t)$ is uniformly convergent.

First, since $|\sin y| \leq|y|$ for all real numbers $y$, we find that

$$
\left|c_{m, n}(t)\right| \leq \frac{2 \pi^{2} x^{2}(n+1)}{m^{2}}
$$

For $m$ larger than $n$ we use the estimate

$$
\sin \left(\frac{\pi x\left(n+\frac{1}{2}+t\right)}{m}\right)=\frac{\pi x\left(n+\frac{1}{2}+t\right)}{m}+O\left(\frac{n^{3}}{m^{3}}\right)
$$


to deduce that

$$
\begin{aligned}
c_{m, n}(t)= & \frac{1}{n+\frac{1}{2}+t}\left(\frac{\pi^{2} x^{2}\left(n+\frac{1}{2}+t\right)^{2}}{m^{2}}+O\left(\frac{n^{4}}{m^{4}}\right)\right) \\
& -\frac{1}{n+\frac{1}{2}-t}\left(\frac{\pi^{2} x^{2}\left(n+\frac{1}{2}-t\right)^{2}}{m^{2}}+O\left(\frac{n^{4}}{m^{4}}\right)\right) \\
= & \frac{\pi^{2} x^{2}\left(n+\frac{1}{2}+t\right)}{m^{2}}-\frac{\pi^{2} x^{2}\left(n+\frac{1}{2}-t\right)}{m^{2}}+O\left(\frac{n^{3}}{m^{4}}\right) \\
= & O\left(\frac{1}{m^{2}}\right)+O\left(\frac{n^{3}}{m^{4}}\right),
\end{aligned}
$$

where here and in the sequel the constants implicit in the $O$-symbols may depend on $x$. For $m \geq n^{3 / 2}$, the estimate above reduces to

$$
c_{m, n}(t)=O\left(\frac{1}{m^{2}}\right)
$$

uniformly for $t \in\left[-\frac{1}{2}, \frac{1}{2}\right]$. It follows that

$$
D_{n}(t)=O\left(\sum_{m \geq n^{3 / 2}} \frac{1}{m^{2}}\right)=O\left(\frac{1}{n^{3 / 2}}\right) .
$$

Hence, the series $\sum_{n=1}^{\infty} D_{n}(t)$ converges uniformly for $t \in\left[-\frac{1}{2}, \frac{1}{2}\right]$.

Next, we examine $A_{n}(t)$. Here we do not have an upper bound for each individual $A_{n}(t)$ to ensure the absolute convergence of $\sum_{n=1}^{\infty} A_{n}(t)$, as we had for $\sum_{n=1}^{\infty} D_{n}(t)$. Instead, we average the terms $A_{n}(t)$ over intervals of the form $\left[N, N+N / \log ^{3} N\right]$ in order to produce enough cancellation to ensure (uniform) convergence of the series. For $m \leq n / \log ^{5} n$, we write $c_{m, n}(t)$ in the form

$$
\begin{aligned}
c_{m, n}(t)= & \frac{1}{n+\frac{1}{2}+t}\left(\frac{1}{2}-\frac{1}{2} \cos \left(\frac{2 \pi x\left(n+\frac{1}{2}+t\right)}{m}\right)\right) \\
& -\frac{1}{n+\frac{1}{2}-t}\left(\frac{1}{2}-\frac{1}{2} \cos \left(\frac{2 \pi x\left(n+\frac{1}{2}-t\right)}{m}\right)\right) \\
= & -\frac{t}{\left(n+\frac{1}{2}\right)^{2}-t^{2}}-\frac{1}{2\left(n+\frac{1}{2}+t\right)} \cos \left(\frac{2 \pi x\left(n+\frac{1}{2}+t\right)}{m}\right) \\
& +\frac{1}{2\left(n+\frac{1}{2}-t\right)} \cos \left(\frac{2 \pi x\left(n+\frac{1}{2}-t\right)}{m}\right) \\
= & \frac{1}{2 n}\left(\cos \left(\frac{2 \pi x\left(n+\frac{1}{2}-t\right)}{m}\right)-\cos \left(\frac{2 \pi x\left(n+\frac{1}{2}+t\right)}{m}\right)\right)+O\left(\frac{1}{n^{2}}\right),
\end{aligned}
$$

where we have used the fact that

$$
\frac{1}{2\left(n+\frac{1}{2} \pm t\right)}-\frac{1}{2 n}=O\left(\frac{1}{n^{2}}\right) .
$$


Using (4.9) in (4.1), we find that

$$
\begin{aligned}
A_{n}(t)= & \frac{1}{2 n} \sum_{1 \leq m \leq n / \log ^{5} n}\left(\cos \left(\frac{2 \pi x\left(n+\frac{1}{2}-t\right)}{m}\right)-\cos \left(\frac{2 \pi x\left(n+\frac{1}{2}+t\right)}{m}\right)\right) \\
& +O\left(\frac{1}{n \log ^{5} n}\right) \\
= & \frac{1}{n} \sum_{1 \leq m \leq n / \log ^{5} n} \sin \left(\frac{2 \pi x t}{m}\right) \sin \left(\frac{\pi x(2 n+1)}{m}\right)+O\left(\frac{1}{n \log ^{5} n}\right),
\end{aligned}
$$

uniformly for $-\frac{1}{2} \leq t \leq \frac{1}{2}$.

We now choose $N$ sufficiently large and average $A_{n}(t)$ over a set of integers from an interval of the form $\left[N, N^{\prime}\right]$, with $N^{\prime} \in\left(N, N+N / \log ^{3} N\right]$, where $N$ and $N^{\prime}$ are both integers. Now,

$$
\sum_{N \leq n \leq N^{\prime}} A_{n}(t)=\sum_{\substack{N \leq n \leq N^{\prime} \\ 1 \leq m \leq n / \log ^{5} n}} \frac{1}{n} \sin \left(\frac{2 \pi x t}{m}\right) \sin \left(\frac{\pi x(2 n+1)}{m}\right)+O\left(\frac{N^{\prime}-N}{N \log ^{5} N}\right)
$$

This further implies that

$$
\begin{aligned}
\left|\sum_{N \leq n \leq N^{\prime}} A_{n}(t)\right| \leq & \sum_{1 \leq m \leq N^{\prime} / \log ^{5} N^{\prime}}\left|\sin \left(\frac{2 \pi x t}{m}\right)\right|\left|\sum_{\substack{N \leq n \leq N^{\prime} \\
n / \log ^{5} n \geq m}} \frac{1}{n} \sin \left(\frac{\pi x(2 n+1)}{m}\right)\right| \\
& +O\left(\frac{1}{\log ^{8} N}\right) \\
\leq & \sum_{1 \leq m \leq 2 N / \log ^{5} N} \frac{\pi x}{m}\left|\sum_{\substack{N \leq n \leq N^{\prime} \\
n / \log ^{5} n \geq m}} \frac{1}{n} \sin \left(\frac{\pi x(2 n+1)}{m}\right)\right|+O\left(\frac{1}{\log ^{8} N}\right)
\end{aligned}
$$

From the definition of $N^{\prime}$, we observe that

$$
\frac{1}{n}=\frac{1}{N}-\frac{n-N}{n N}=\frac{1}{N}+O\left(\frac{1}{N \log ^{3} N}\right)
$$


Hence,

$$
\begin{aligned}
\left|\sum_{N \leq n \leq N^{\prime}} A_{n}(t)\right| \leq & \sum_{1 \leq m \leq 2 N / \log ^{5} N} \frac{\pi x}{m}\left(\frac{1}{N}\left|\sum_{\substack{N \leq n \leq N^{\prime} \\
n / \log ^{5} n \geq m}} \sin \left(\frac{\pi x(2 n+1)}{m}\right)\right|+O\left(\frac{N^{\prime}-N}{N \log ^{3} N}\right)\right) \\
& +O\left(\frac{1}{\log ^{8} N}\right) \\
= & \frac{\pi x}{N} \sum_{1 \leq m \leq 2 N / \log ^{5} N} \frac{1}{m}\left|\sum_{\substack{N \leq n \leq N^{\prime} \\
n / \log ^{5} n \geq m}} \sin \left(\frac{\pi x(2 n+1)}{m}\right)\right|+O\left(\frac{1}{\log ^{5} N}\right) .
\end{aligned}
$$

It should be remarked that on the right side of (4.11) we need the error term to be that small; more precisely, an error term of the form

$$
O\left(\frac{1}{\log ^{4} N}\right)
$$

would be insufficient. Next, the inner sum on the right side of (4.11) has the form

$$
S_{m}:=\sum_{N_{m}^{\prime \prime} \leq n \leq N^{\prime}} \sin \left(\frac{\pi x(2 n+1)}{m}\right),
$$

for some $N_{m}^{\prime \prime}$ that depends on $m$. We distinguish two cases. If $x / m$ is an integer, then clearly $S_{m}=0$, and so such values of $m$, if there are any, can be excluded from the summation on the right side of (4.11). On the other hand, if $x / m$ is not an integer, then $e^{2 \pi i x / m}-1 \neq 0$, and we write

$$
\begin{aligned}
S_{m} & =\frac{1}{2 i} \sum_{N_{m}^{\prime \prime} \leq n \leq N^{\prime}}\left(e^{\pi i x(2 n+1) / m}-e^{-\pi i x(2 n+1) / m}\right) \\
& =\frac{e^{\pi i x / m}}{2 i} \sum_{N_{m}^{\prime \prime} \leq n \leq N^{\prime}} e^{2 \pi i x n / m}-\frac{e^{-\pi i x / m}}{2 i} \sum_{N_{m}^{\prime \prime} \leq n \leq N^{\prime}} e^{-2 \pi i x n / m} .
\end{aligned}
$$

Therefore,

$$
\begin{aligned}
\left|S_{m}\right| & \leq\left|\sum_{N_{m}^{\prime \prime} \leq n \leq N^{\prime}} e^{2 \pi i x n / m}\right|=\frac{\left|e^{2 \pi i x N_{m}^{\prime \prime} / m}-e^{2 \pi i x\left(N^{\prime}+1\right) / m}\right|}{\left|e^{2 \pi i x / m}-1\right|} \\
& \leq \frac{2}{\left|e^{2 \pi i x / m}-1\right|}=\frac{2}{\left|e^{\pi i x / m}-e^{-\pi i x / m}\right|}=\frac{1}{|\sin (\pi x / m)|} .
\end{aligned}
$$

If we consider the real numbers $x / m, 1 \leq m \leq 2 x$, and select the one that is closest to an integer, without being an integer itself, and if we let $\delta(x)$ denote the distance from this number to the closest integer, then $\delta(x)>0$, and $\delta(x)$ depends only on $x$. Thus, 
for any integer $m$ such that $1 \leq m \leq 2 x$, by (4.12), we deduce that

$$
\left|S_{m}\right| \leq \frac{1}{|\sin (\pi x / m)|}=O\left(\frac{1}{\delta(x)}\right)=O(1)
$$

since $x$ is fixed. For any $m$ with $m>2 x$, clearly, $\pi x / m \in\left(0, \frac{1}{2} \pi\right)$, and $\operatorname{so} \sin (\pi x / m) \geq$ $c_{0} \pi x / m$, for some absolute constant $c_{0}>0$. Thus, for such $m$, by (4.12),

$$
\left|S_{m}\right| \leq \frac{m}{c_{0} \pi x}+O(m)
$$

Employing (4.13) and (4.14) in (4.11), we deduce that

$$
\begin{aligned}
\left|\sum_{N \leq n \leq N^{\prime}} A_{n}(t)\right| & =O\left(\frac{1}{N} \sum_{1 \leq m \leq 2 N / \log ^{5} N} \frac{1}{m} \cdot m\right)+O\left(\frac{1}{\log ^{5} N}\right) \\
& =O\left(\frac{1}{\log ^{5} N}\right),
\end{aligned}
$$

uniformly in $t \in\left[-\frac{1}{2}, \frac{1}{2}\right]$.

We are now ready to show that the series $\sum_{n=1}^{\infty} A_{n}(t)$ converges uniformly for $t \in$ $\left[-\frac{1}{2}, \frac{1}{2}\right]$. As an intermediate step, let us choose two large positive numbers $N_{1}$ and $N_{2}$ such that $N_{1} \leq N_{2} \leq 2 N_{1}$. We seek an upper bound for the sum $\sum_{N_{1} \leq n \leq N_{2}} A_{n}(t)$. In order to derive such a bound, we divide the interval $\left[N_{1}, N_{2}\right]$ into subintervals of the form $\left[N, N^{\prime}\right]$, with $N \leq N^{\prime} \leq N+N / \log ^{3} N$, and for each of them we apply the upper bound from (4.15). Clearly, since $N_{2} \in\left[N_{1}, 2 N_{1}\right]$, we obtain a partition of the subinterval $\left[N_{1}, N_{2}\right]$, with the number of subintervals bounded by $\log ^{3} N_{1}$. We then deduce from (4.15) that

$$
\left|\sum_{N_{1} \leq n \leq N_{2}} A_{n}(t)\right|=O\left(\frac{1}{\log ^{2} N_{1}}\right),
$$

uniformly for $-\frac{1}{2} \leq t \leq \frac{1}{2}$. In particular, if we apply (4.16) with $N_{1}=2^{k}$ and $N_{2}=2^{k+1}$, for some positive integer $k$, we find that

$$
\left|\sum_{2^{k} \leq n \leq 2^{k+1}} A_{n}(t)\right|=O\left(\frac{1}{k^{2}}\right),
$$

uniformly for $t \in\left[-\frac{1}{2}, \frac{1}{2}\right]$. Lastly, if $N$ and $N^{\prime}$ are arbitrary positive integers such that $N<N^{\prime}$, we choose $k$ and $k^{\prime}$ such that $2^{k} \leq N \leq 2^{k+1}$ and $2^{k^{\prime}} \leq N^{\prime} \leq 2^{k^{\prime}+1}$, and then apply (4.16) and (4.17), as appropriate, to the intervals

$$
\left[N, 2^{k+1}\right],\left[2^{k+1}, 2^{k+2}\right], \ldots,\left[2^{k^{\prime}-1}, 2^{k^{\prime}}\right],\left[2^{k^{\prime}}, N^{\prime}\right] .
$$

We therefore conclude that

$$
\left|\sum_{N \leq n \leq N^{\prime}} A_{n}(t)\right|=O\left(\sum_{k \leq \ell \leq k^{\prime}} \frac{1}{\ell^{2}}\right)=O\left(\frac{1}{k}\right),
$$


uniformly for $-\frac{1}{2} \leq t \leq \frac{1}{2}$. The estimate (4.18) implies, by Cauchy's criterion, that the series of functions $\sum_{n=1}^{\infty} A_{n}(t)$ is uniformly convergent for $-\frac{1}{2} \leq t \leq \frac{1}{2}$.

Next, we turn to the series $\sum_{n=1}^{\infty} C_{n}(t)$. As was the case with the functions $D_{n}(t)$, the functions $C_{n}(t)$ are small enough to ensure the desired absolute and uniform convergence. In order to show this, we first derive some sharper estimates for the terms $c_{m, n}(t)$ in the corresponding range $n \log ^{2} n<m<n^{3 / 2}$. First, since

$$
\sin ^{2}\left(\frac{\pi x\left(n+\frac{1}{2} \pm t\right)}{m}\right)=O\left(\frac{n^{2}}{m^{2}}\right)
$$

it follows that

$$
\begin{aligned}
\sum_{n \log ^{2} n<m<n^{3 / 2}}\left(\sin ^{2}\left(\frac{\pi x\left(n+\frac{1}{2}+t\right)}{m}\right)\right. & \left.+\sin ^{2}\left(\frac{\pi x\left(n+\frac{1}{2}-t\right)}{m}\right)\right) \\
= & O\left(\sum_{n \log ^{2} n<m<n^{3 / 2}} \frac{n^{2}}{m^{2}}\right)=O\left(\frac{n}{\log ^{2} n}\right) .
\end{aligned}
$$

Also,

$$
\frac{1}{n+\frac{1}{2} \pm t}=\frac{1}{n}+O\left(\frac{1}{n^{2}}\right)
$$

By (4.19), (4.20), and (4.5), we then derive that

$$
\begin{aligned}
c_{m, n}(t)= & \frac{1}{n} \sum_{n \log ^{2} n<m<n^{3 / 2}}\left(\sin ^{2}\left(\frac{\pi x\left(n+\frac{1}{2}+t\right)}{m}\right)-\sin ^{2}\left(\frac{\pi x\left(n+\frac{1}{2}-t\right)}{m}\right)\right) \\
& +O\left(\frac{1}{n^{2}} \sum_{n \log ^{2} n<m<n^{3 / 2}}\left(\sin ^{2}\left(\frac{\pi x\left(n+\frac{1}{2}+t\right)}{m}\right)+\sin ^{2}\left(\frac{\pi x\left(n+\frac{1}{2}-t\right)}{m}\right)\right)\right) \\
= & \frac{1}{n} \sum_{n \log ^{2} n<m<n^{3 / 2}} \sin \left(\frac{2 \pi x t}{m}\right) \sin \left(\frac{\pi x(2 n+1)}{m}\right)+O\left(\frac{1}{n \log ^{2} n}\right) \\
= & O\left(\frac{1}{n} \sum_{n \log ^{2} n<m<n^{3 / 2}} \frac{1}{m} \cdot \frac{n}{m}\right)+O\left(\frac{1}{n \log ^{2} n}\right) \\
= & O\left(\sum_{n \log ^{2} n<m<n^{3 / 2}} \frac{1}{m^{2}}\right)+O\left(\frac{1}{n \log ^{2} n}\right)=O\left(\frac{1}{n \log ^{2} n}\right),
\end{aligned}
$$

uniformly for $-\frac{1}{2} \leq t \leq \frac{1}{2}$. It follows that the series $\sum_{n=1}^{\infty} C_{n}(t)$ converges absolutely and uniformly for $-\frac{1}{2} \leq t \leq \frac{1}{2}$.

Lastly, we turn to the sum $\sum_{n=1}^{\infty} B_{n}(t)$. For $n / \log ^{5} n<m \leq n \log ^{2} n$, we use the estimates

$$
\frac{1}{n+\frac{1}{2} \pm t}=\frac{1}{n+\frac{1}{2}} \mp \frac{t}{\left(n+\frac{1}{2}\right)^{2}}+O\left(\frac{1}{n^{3}}\right)=\frac{1}{n+\frac{1}{2}} \mp \frac{t}{n^{2}}+O\left(\frac{1}{n^{3}}\right),
$$




$$
\sin ^{2}\left(\frac{\pi x\left(n+\frac{1}{2} \pm t\right)}{m}\right)=\sin ^{2}\left(\frac{\pi x n}{m}\right)+O\left(\frac{1}{m}\right)=\sin ^{2}\left(\frac{\pi x n}{m}\right)+O\left(\frac{\log ^{5} n}{n}\right)
$$

and

$$
\sin ^{2}\left(\frac{\pi x\left(n+\frac{1}{2}+t\right)}{m}\right)-\sin ^{2}\left(\frac{\pi x\left(n+\frac{1}{2}-t\right)}{m}\right)=\sin \left(\frac{2 \pi x t}{m}\right) \sin \left(\frac{\pi x(2 n+1)}{m}\right),
$$

in the definition of $B_{n}(t)$ to derive that

$$
\begin{aligned}
B_{n}(t)= & \sum_{n / \log ^{5} n<m \leq n \log ^{2} n}\left(\left(\frac{1}{n+\frac{1}{2}}-\frac{t}{n^{2}}\right) \sin ^{2}\left(\frac{\pi x\left(n+\frac{1}{2}+t\right)}{m}\right)\right. \\
& \left.-\left(\frac{1}{n+\frac{1}{2}}+\frac{t}{n^{2}}\right) \sin ^{2}\left(\frac{\pi x\left(n+\frac{1}{2}-t\right)}{m}\right)+O\left(\frac{1}{n^{3}}\right)\right) \\
= & \frac{1}{n+\frac{1}{2}} \sum_{n / \log ^{5} n<m \leq n \log ^{2} n} \sin \left(\frac{2 \pi x t}{m}\right) \sin \left(\frac{\pi x(2 n+1)}{m}\right) \\
& -\frac{t}{n^{2}} \sum_{n / \log ^{5} n<m \leq n \log ^{2} n}\left(2 \sin ^{2}\left(\frac{\pi x n}{m}\right)+O\left(\frac{\log ^{5} n}{n}\right)\right)+O\left(\frac{n \log ^{2} n}{n^{3}}\right) \\
= & \frac{1}{n+\frac{1}{2}} \sum_{n / \log ^{5}} \sum_{n<m \leq n} \sin \left(\frac{2 \pi x t}{m}\right) \sin \left(\frac{\pi x(2 n+1)}{m}\right) \\
& -\frac{2 t}{n^{2}} \sum_{n / \log ^{5} n} \sin _{n<m \leq n \log ^{2} n}\left(\frac{\pi x n}{m}\right)+O\left(\frac{\log ^{7} n}{n^{2}}\right) .
\end{aligned}
$$

We would like to approximate each of the two sums on the right side of (4.22) by an appropriate integral. Before we do this, we slightly modify the first sum. On the given range of $m$, we observe that

$$
\sin \left(\frac{2 \pi x t}{m}\right)=\frac{2 \pi x t}{m}+O\left(\frac{1}{m^{3}}\right)=\frac{2 \pi x t}{m}+O\left(\frac{\log ^{15} n}{n^{3}}\right)
$$

and so the contribution of the error term from (4.23) in (4.22) is

$$
O\left(\frac{1}{n} \sum_{n / \log ^{5}} \sum_{n<m \leq n \log ^{2} n} \frac{\log ^{15} n}{n^{3}}\right)=O\left(\frac{\log ^{17} n}{n^{3}}\right) .
$$

Hence, from (4.22),

$$
\begin{aligned}
B_{n}(t)= & \frac{2 \pi x t}{n+\frac{1}{2}} \sum_{n / \log ^{5}} \sum_{n<m \leq n \log ^{2} n} \frac{1}{m} \sin \left(\frac{\pi x(2 n+1)}{m}\right) \\
& -\frac{2 t}{n^{2}} \sum_{n / \log ^{5}} \sum_{n<m \leq n \log ^{2} n} \sin ^{2}\left(\frac{\pi x n}{m}\right)+O\left(\frac{\log ^{7} n}{n^{2}}\right) .
\end{aligned}
$$


Next, we apply the Euler-Maclaurin summation formula to the function

$$
g(y):=\frac{1}{y} \sin \left(\frac{\pi x(2 n+1)}{y}\right)
$$

to find that

$$
\begin{array}{r}
\sum_{n / \log ^{5} n<m \leq n \log ^{2} n} \frac{1}{m} \sin \left(\frac{\pi x(2 n+1)}{m}\right)=\int_{n / \log ^{5} n}^{n \log ^{2} n} g(y) d y+\int_{n / \log ^{5} n}^{n \log ^{2} n}\{y\} g^{\prime}(y) d y \\
-\left\{n \log ^{2} n\right\} g\left(n \log ^{2} n\right)+\left\{\frac{n}{\log ^{5} n}\right\} g\left(\frac{n}{\log ^{5} n}\right),
\end{array}
$$

where $\{x\}$ denotes the fractional part of $x$. The last two terms on the right side of (4.25) are each bounded by $\left(\log ^{5} n\right) / n$. Also, for $n / \log ^{5} n \leq y \leq n \log ^{2} n$, we see that

$$
\begin{aligned}
g^{\prime}(y) & =-\frac{1}{y^{2}} \sin \left(\frac{\pi x(2 n+1)}{y}\right)-\frac{\pi x(2 n+1)}{y^{3}} \cos \left(\frac{\pi x(2 n+1)}{y}\right) \\
& =O\left(\frac{1}{y^{2}}+\frac{n}{y^{3}}\right)=O\left(\frac{\log ^{15} n}{n^{2}}\right),
\end{aligned}
$$

and hence

$$
\int_{n / \log ^{5} n}^{n \log ^{2} n}\{y\} g^{\prime}(y) d y=O\left(\frac{\log ^{17} n}{n}\right) .
$$

Using this last estimate in (4.25), we conclude that

$$
\begin{aligned}
\sum_{n / \log ^{5} n<m \leq n \log ^{2} n} & \frac{1}{m} \sin \left(\frac{\pi x(2 n+1)}{m}\right)=\int_{n / \log ^{5} n}^{n \log ^{2} n} \frac{1}{y} \sin \left(\frac{\pi x(2 n+1)}{y}\right) d y+O\left(\frac{\log ^{17} n}{n}\right) \\
& =\int_{n / \log ^{5} n}^{n \log ^{2} n} \frac{1}{y}\left(\sin \left(\frac{2 \pi x n}{y}\right)+O\left(\frac{1}{y}\right)\right) d y+O\left(\frac{\log ^{17} n}{n}\right) \\
& =\int_{n / \log ^{5} n}^{n \log ^{2} n} \frac{1}{y} \sin \left(\frac{2 \pi x n}{y}\right) d y+O\left(\frac{\log ^{17} n}{n}\right) .
\end{aligned}
$$

On the far right side of (4.26), make the change of variable $u=2 \pi x n / y$ to find that

$$
\sum_{n / \log ^{5} n<m \leq n \log ^{2} n} \frac{1}{m} \sin \left(\frac{\pi x(2 n+1)}{m}\right)=\int_{2 \pi x / \log ^{2} n}^{2 \pi x \log ^{5} n} \frac{\sin u}{u} d u+O\left(\frac{\log ^{17} n}{n}\right) .
$$

Recall that

Clearly,

$$
\int_{0}^{\infty} \frac{\sin u}{u} d u=\frac{\pi}{2}
$$

$$
\int_{0}^{2 \pi x / \log ^{2} n} \frac{\sin u}{u} d u=O\left(\frac{1}{\log ^{2} n}\right) .
$$

Also, by an integration by parts, we easily see that

$$
\int_{2 \pi x \log ^{5} n}^{\infty} \frac{\sin u}{u} d u=O\left(\frac{1}{\log ^{5} n}\right) .
$$


Using these last three equalities in (4.27), we find that

$$
\sum_{n / \log ^{5}} \frac{1}{m<m \leq n} \sin \left(\frac{\pi x(2 n+1)}{m}\right)=\frac{\pi}{2}+O\left(\frac{1}{\log ^{2} n}\right) .
$$

Inserting this last result in (4.24), we arrive at

$$
\begin{aligned}
B_{n}(t) & =\frac{\pi^{2} x t}{n+\frac{1}{2}}-\frac{2 t}{n^{2}} \sum_{n / \log ^{5}} \sin _{n<m \leq n} \operatorname{sog}^{2} n \\
& =\frac{\pi^{2} x t}{n}-\frac{2 t}{n^{2}} \sum_{n / \log ^{5}} \sum_{n<m \leq n} \sin ^{2}\left(\frac{\pi x n}{m}\right)+O\left(\frac{1}{n \log ^{2} n}\right)
\end{aligned}
$$

Next, we apply the Euler-Maclaurin summation formula to the function

$$
h(y):=\sin ^{2}\left(\frac{\pi x n}{y}\right)
$$

to obtain the equality

$$
\begin{aligned}
\sum_{n / \log ^{5}} \sin ^{2}\left(\frac{\pi x n}{m}\right) & =\int_{n / \log ^{5} n}^{n \log ^{2} n} h(y) d y+\int_{n / \log ^{5} n}^{n \log ^{2} n}\{y\} h^{\prime}(y) d y \\
& -\left\{n \log ^{2} n\right\} h\left(n \log ^{2} n\right)+\left\{\frac{n}{\log ^{5} n}\right\} h\left(\frac{n}{\log ^{5} n}\right) .
\end{aligned}
$$

Each of the last two terms on the right side of (4.29) is $O(1)$. Also, for $n / \log ^{5} n<y \leq$ $n \log ^{2} n$,

$$
h^{\prime}(y)=-\frac{2 \pi x n}{y^{2}} \sin \left(\frac{\pi x n}{y}\right) \cos \left(\frac{\pi x n}{y}\right)=O\left(\frac{n}{y^{2}}\right)=O\left(\frac{\log ^{10} n}{n}\right)
$$

so that

$$
\int_{n / \log ^{5} n}^{n \log ^{2} n}\{y\} h^{\prime}(y) d y=O\left(\log ^{12} n\right)
$$

Thus,

$$
\begin{aligned}
\sum_{n / \log ^{5} n<m \leq n \log ^{2} n} \sin ^{2}\left(\frac{\pi x n}{m}\right) & =\int_{n / \log ^{5} n}^{n \log ^{2} n} \sin ^{2}\left(\frac{\pi x n}{y}\right) d y+O\left(\log ^{12} n\right) \\
& =\pi x n \int_{\pi x / \log ^{2} n}^{\pi x \log ^{5} n} \frac{\sin ^{2} u}{u^{2}} d u+O\left(\log ^{12} n\right)
\end{aligned}
$$

upon making the change of variable $u=\pi x n / y$. Clearly,

$$
\int_{0}^{\pi x / \log ^{2} n} \frac{\sin ^{2} u}{u^{2}} d u=O\left(\frac{1}{\log ^{2} n}\right)
$$

and

$$
\int_{\pi x \log ^{5} n}^{\infty} \frac{\sin ^{2} u}{u^{2}} d u=O\left(\frac{1}{\log ^{5} n}\right)
$$


Therefore, from (4.30)

$$
\sum_{n / \log ^{5}} \sin _{n<m \leq n}^{2}\left(\frac{\pi x n}{m}\right)=\pi x n \int_{0}^{\infty} \frac{\sin ^{2} u}{u^{2}} d u+O\left(\frac{n}{\log ^{2} n}\right) .
$$

Lastly, using the result above in (4.28), we conclude that

$$
\begin{aligned}
B_{n}(t) & =\frac{\pi^{2} x t}{n}-\frac{2 t}{n^{2}}\left(\pi x n \int_{0}^{\infty} \frac{\sin ^{2} u}{u^{2}} d u+O\left(\frac{n}{\log ^{2} n}\right)\right)+O\left(\frac{1}{n \log ^{2} n}\right) \\
& =\frac{\pi x t}{n}\left(\pi-2 \int_{0}^{\infty} \frac{\sin ^{2} u}{u^{2}} d u\right)+O\left(\frac{1}{n \log ^{2} n}\right) \\
& =O\left(\frac{1}{n \log ^{2} n}\right),
\end{aligned}
$$

uniformly for $-\frac{1}{2} \leq t \leq \frac{1}{2}$, upon using the evaluation

$$
\int_{0}^{\infty} \frac{\sin ^{2} u}{u^{2}} d u=\frac{\pi}{2}
$$

It follows that the series $B_{n}(t)$ converges absolutely and uniformly for $-\frac{1}{2} \leq t \leq \frac{1}{2}$.

In conclusion,

$$
\sum_{n=0}^{\infty} f_{n}(t)=\sum_{n=0}^{\infty}\left(A_{n}(t)+B_{n}(t)+C_{n}(t)+D_{n}(t)\right)
$$

converges uniformly for $-\frac{1}{2} \leq t \leq \frac{1}{2}$, as claimed earlier, and so, for any real number $x>0$, the function $f(t)=\sum_{n=0}^{\infty} f_{n}(t)$ is well defined, and it is continuous on $\left[-\frac{1}{2}, \frac{1}{2}\right]$.

\section{Identifying the Fourier COefFicients}

Having established the convergence of the double series in (3.13), we now focus our attention on proving the identity (3.13) itself. For fixed $x>0$, define the function $\tilde{f}$ by

$$
\tilde{f}(t):=\sum_{1 \leq n \leq x}(-1)^{n} F\left(\frac{x}{n}\right) \sin (2 \pi n t)+\pi x t, \quad t \in\left[-\frac{1}{2}, \frac{1}{2}\right] .
$$

Then (3.17) reduces to

$$
\frac{1}{\pi} f(t)=\tilde{f}(t), \quad t \in\left[-\frac{1}{2}, \frac{1}{2}\right]
$$

In order to prove (5.2), it is enough to show that for any integer $k$,

$$
\frac{1}{\pi} \int_{-1 / 2}^{1 / 2} f(t) e^{2 \pi i k t} d t=\int_{-1 / 2}^{1 / 2} \tilde{f}(t) e^{2 \pi i k t} d t .
$$

With the use of (3.18), (5.3) takes the form

$$
\sum_{n=0}^{\infty} \int_{-1 / 2}^{1 / 2} f_{n}(t) e^{2 \pi i k t} d t=\pi \int_{-1 / 2}^{1 / 2} \tilde{f}(t) e^{2 \pi i k t} d t
$$


Since $f(t)$ and $f_{n}(t), n \geq 0$, are odd functions of $t,(5.4)$ will be valid provided that we can show that, for every integer $k \geq 1$,

$$
\sum_{n=0}^{\infty} \int_{-1 / 2}^{1 / 2} f_{n}(t) \sin (2 \pi k t) d t=\pi \int_{-1 / 2}^{1 / 2} \tilde{f}(t) \sin (2 \pi k t) d t
$$

Now, by (5.1),

$$
\begin{aligned}
\int_{-1 / 2}^{1 / 2} \tilde{f}(t) \sin (2 \pi k t) d t & =\pi x \int_{-1 / 2}^{1 / 2} t \sin (2 \pi k t) d t+(-1)^{k} F\left(\frac{x}{k}\right) \int_{-1 / 2}^{1 / 2} \sin ^{2}(2 \pi k t) d t \\
& =(-1)^{k+1} \frac{x}{2 k}+(-1)^{k} \frac{1}{2} F\left(\frac{x}{k}\right) .
\end{aligned}
$$

Thus, (5.5) reduces to

$$
\sum_{n=0}^{\infty} \int_{-1 / 2}^{1 / 2} f_{n}(t) \sin (2 \pi k t) d t=(-1)^{k+1} \frac{\pi x}{2 k}+(-1)^{k} \frac{\pi}{2} F\left(\frac{x}{k}\right) .
$$

In the sequel, both $x$ and $k$ will be fixed. In the estimates below, constants implied by the $O$-symbols depend (at most) on $x$ and $k$.

For any positive integer $N$, let

$$
I_{N}:=\sum_{n=0}^{N-1} \int_{-1 / 2}^{1 / 2} f_{n}(t) \sin (2 \pi k t) d t
$$

so that (5.6) is equivalent to

$$
\lim _{N \rightarrow \infty} I_{N}=(-1)^{k+1} \frac{\pi x}{2 k}+(-1)^{k} \frac{\pi}{2} F\left(\frac{x}{k}\right) .
$$

Choosing a large positive integer $N$ and using the definition (4.5), write $I_{N}$ in the form

$$
I_{N}=\sum_{n=0}^{N-1} \int_{-1 / 2}^{1 / 2} \sin (2 \pi k t) \sum_{m=1}^{\infty} c_{m, n}(t) d t
$$

where $c_{m, n}(t)$ is defined by (4.5). Since for each fixed $n$, the series $\sum_{m=1}^{\infty} c_{m, n}(t)$ converges absolutely and uniformly for $-\frac{1}{2} \leq t \leq \frac{1}{2}$, we may interchange summation and integration, and then interchange the order of summation, to put $I_{N}$ in the form

$$
I_{N}=\sum_{n=0}^{N-1} \sum_{m=1}^{\infty} \int_{-1 / 2}^{1 / 2} \sin (2 \pi k t) c_{m, n}(t) d t=\sum_{m=1}^{\infty} \sum_{n=0}^{N-1} \int_{-1 / 2}^{1 / 2} \sin (2 \pi k t) c_{m, n}(t) d t .
$$

For each fixed $m$, we find that

$$
\begin{aligned}
\sum_{n=0}^{N-1} \int_{-1 / 2}^{1 / 2} \sin (2 \pi k t) c_{m, n}(t) d t= & \sum_{n=0}^{N-1} \int_{-1 / 2}^{1 / 2} \frac{\sin (2 \pi k t)}{n+\frac{1}{2}+t} \sin ^{2}\left(\frac{\pi x\left(n+\frac{1}{2}+t\right)}{m}\right) d t \\
& -\sum_{n=0}^{N-1} \int_{-1 / 2}^{1 / 2} \frac{\sin (2 \pi k t)}{n+\frac{1}{2}-t} \sin ^{2}\left(\frac{\pi x\left(n+\frac{1}{2}-t\right)}{m}\right) d t
\end{aligned}
$$




$$
\begin{aligned}
= & \sum_{n=0}^{N-1} \int_{n}^{n+1} \frac{\sin \left(2 \pi k\left(u-n-\frac{1}{2}\right)\right)}{u} \sin ^{2}\left(\frac{\pi x u}{m}\right) d u \\
& +\sum_{n=0}^{N-1} \int_{-n-1}^{-n} \frac{\sin \left(2 \pi k\left(u+n+\frac{1}{2}\right)\right)}{u} \sin ^{2}\left(\frac{\pi x u}{m}\right) d u \\
= & (-1)^{k} \int_{-N}^{N} \frac{\sin (2 \pi k u)}{u} \sin ^{2}\left(\frac{\pi x u}{m}\right) d u .
\end{aligned}
$$

For each pair of positive integers $m, N$, set

$$
I_{N, m}:=\int_{-N}^{N} \frac{\sin (2 \pi k u)}{u} \sin ^{2}\left(\frac{\pi x u}{m}\right) d u .
$$

Then, from (5.10)-(5.12),

$$
I_{N}=(-1)^{k} \sum_{m=1}^{\infty} I_{N, m} .
$$

Inserting (5.13) into (5.8), we see that it remains to prove the equality

$$
\lim _{N \rightarrow \infty} \sum_{m=1}^{\infty} I_{N, m}=\frac{\pi}{2} F\left(\frac{x}{k}\right)-\frac{\pi x}{2 k} .
$$

For $m>N$, we estimate $I_{N, m}$ by integrating by parts on the right side of (5.12) and finding that

$$
\begin{aligned}
I_{N, m}= & -\left.\frac{\cos (2 \pi k u)}{2 \pi k} \cdot \frac{1}{u} \sin ^{2}\left(\frac{\pi x u}{m}\right)\right|_{-N} ^{N} \\
& +\int_{-N}^{N} \frac{\cos (2 \pi k u)}{2 \pi k}\left(-\frac{1}{u^{2}} \sin ^{2}\left(\frac{\pi x u}{m}\right)+\frac{\pi x}{m u} \sin \left(\frac{2 \pi x u}{m}\right)\right) d u \\
= & -\frac{1}{\pi k N} \sin ^{2}\left(\frac{\pi x N}{m}\right)-\frac{1}{2 \pi k} \int_{-N}^{N} \frac{\cos (2 \pi k u)}{u^{2}} \sin ^{2}\left(\frac{\pi x u}{m}\right) d u \\
& +\frac{x}{2 k m} \int_{-N}^{N} \frac{\cos (2 \pi k u)}{u} \sin \left(\frac{2 \pi x u}{m}\right) d u .
\end{aligned}
$$

On the right side of $(5.15)$, we use the estimates

$$
\begin{aligned}
\sin ^{2}\left(\frac{\pi x N}{m}\right) & =O\left(\frac{N^{2}}{m^{2}}\right), \\
\frac{1}{u^{2}} \sin ^{2}\left(\frac{\pi x u}{m}\right) & =O\left(\frac{1}{m^{2}}\right),
\end{aligned}
$$

and

$$
\frac{1}{u} \sin \left(\frac{2 \pi x u}{m}\right)=O\left(\frac{1}{m}\right)
$$


uniformly in $u$, in order to conclude that

$$
I_{N, m}=O\left(\frac{N}{m^{2}}\right) \text {. }
$$

Now let $L_{N}$ and $M_{N}$ be integers such that $1<L_{N}<N<M_{N}$, where the values of $L_{N}$ and $M_{N}$ will be chosen later. By (5.16),

$$
\sum_{m>M_{N}} I_{N, m}=O\left(\sum_{m>M_{N}} \frac{N}{m^{2}}\right)=O\left(\frac{N}{M_{N}}\right) .
$$

We shall let $N \rightarrow \infty$ and choose $M_{N}$ so that $N / M_{N} \rightarrow 0$. Hence, from (5.17), it will follow that

$$
\lim _{N \rightarrow \infty} \sum_{m>M_{N}} I_{N, m}=0
$$

and so (5.14) reduces to

$$
\lim _{N \rightarrow \infty} \sum_{1 \leq m \leq M_{N}} I_{N, m}=\frac{\pi}{2} F\left(\frac{x}{k}\right)-\frac{\pi x}{2 k} .
$$

In the range $L_{N}<m \leq M_{N}$, we use (5.15), sum on $m$, and interchange summation and integration to arrive at

$$
\begin{aligned}
\sum_{L_{N}<m \leq M_{N}} I_{N, m}= & -\frac{1}{\pi k N} \sum_{L_{N}<m \leq M_{N}} \sin ^{2}\left(\frac{\pi x N}{m}\right) \\
& -\frac{1}{2 \pi k} \int_{-N}^{N} \frac{\cos (2 \pi k u)}{u^{2}} \sum_{L_{N}<m \leq M_{N}} \sin ^{2}\left(\frac{\pi x u}{m}\right) d u \\
& +\frac{x}{2 k} \int_{-N}^{N} \frac{\cos (2 \pi k u)}{u} \sum_{L_{N}<m \leq M_{N}} \frac{1}{m} \sin \left(\frac{2 \pi x u}{m}\right) d u .
\end{aligned}
$$

In order to estimate the first sum on the right side of (5.19), we apply the EulerMaclaurin summation formula to the function

$$
h_{N}(y):=\sin ^{2}\left(\frac{\pi x N}{y}\right) .
$$

Recalling that $L_{N}$ and $M_{N}$ are positive integers, we therefore deduce that

$$
\sum_{L_{N}<m \leq M_{N}} \sin ^{2}\left(\frac{\pi x N}{m}\right)=\int_{L_{N}}^{M_{N}} \sin ^{2}\left(\frac{\pi x N}{y}\right) d y+\int_{L_{N}}^{M_{N}}\{y\} h_{N}^{\prime}(y) d y .
$$

Here,

$$
h_{N}^{\prime}(y)=-\frac{2 \pi x N}{y^{2}} \sin \left(\frac{\pi x N}{y}\right) \cos \left(\frac{\pi x N}{y}\right)=O\left(\frac{N}{y^{2}}\right)
$$

and hence

$$
\int_{L_{N}}^{M_{N}}\{y\} h_{N}^{\prime}(y) d y=O\left(N \int_{L_{N}}^{M_{N}} \frac{1}{y^{2}} d y\right)=O\left(\frac{N}{L_{N}}\right)
$$


Also,

$$
\begin{aligned}
\int_{L_{N}}^{M_{N}} \sin ^{2}\left(\frac{\pi x N}{y}\right) d y & =\pi x N \int_{\pi x N / M_{N}}^{\pi x N / L_{N}} \frac{\sin ^{2} u}{u^{2}} d u \\
& =\pi x N\left(\int_{0}^{\infty}-\int_{0}^{\pi x N / M_{N}}-\int_{\pi x N / L_{N}}^{\infty} \frac{\sin ^{2} u}{u^{2}} d u\right. \\
& =\pi x N\left(\frac{\pi}{2}+O\left(\frac{N}{M_{N}}\right)+O\left(\frac{L_{N}}{N}\right)\right) .
\end{aligned}
$$

Using (5.21) and (5.22) in (5.20), we see that

$$
\sum_{L_{N}<m \leq M_{N}} \sin ^{2}\left(\frac{\pi x N}{m}\right)=\frac{\pi^{2} x N}{2}+O\left(\frac{N^{2}}{M_{N}}\right)+O\left(L_{N}\right)+O\left(\frac{N}{L_{N}}\right) .
$$

Next, for each fixed $u \in[-N, N]$, we estimate the sum inside the first integral on the right side of (5.19) by applying the Euler-Maclaurin summation formula to

$$
h_{u}(y):=\sin ^{2}\left(\frac{\pi x u}{y}\right) .
$$

Accordingly,

$$
\sum_{L_{N}<m \leq M_{N}} \sin ^{2}\left(\frac{\pi x u}{m}\right)=\int_{L_{N}}^{M_{N}} \sin ^{2}\left(\frac{\pi x u}{y}\right) d y+\int_{L_{N}}^{M_{N}}\{y\} h_{u}^{\prime}(y) d y .
$$

Since we are going to apply (5.24) for both small and large $u$, we need to exercise care in estimating $h_{u}^{\prime}(y)$. To that end,

$$
h_{u}^{\prime}(y)=-\frac{2 \pi x u}{y^{2}} \sin \left(\frac{\pi x u}{y}\right) \cos \left(\frac{\pi x u}{y}\right)=O\left(\frac{|u|}{y^{2}} \min \left\{1, \frac{|u|}{y}\right\}\right) .
$$

Hence,

$$
\begin{aligned}
\int_{L_{N}}^{M_{N}}\{y\} h_{u}^{\prime}(y) d y & =\int_{L_{N}}^{\max \left\{L_{N},|u|\right\}}\{y\} h_{u}^{\prime}(y) d y+\int_{\max \left\{L_{N},|u|\right\}}^{M_{N}}\{y\} h_{u}^{\prime}(y) d y \\
& =O\left(\int_{L_{N}}^{\max \left\{L_{N},|u|\right\}} \frac{|u|}{y^{2}} d y\right)+O\left(\int_{\max \left\{L_{N},|u|\right\}}^{M_{N}} \frac{|u|^{2}}{y^{3}} d y\right) \\
& =O\left(|u|\left(\frac{1}{L_{N}}-\frac{1}{\max \left\{L_{N},|u|\right\}}\right)\right)+O\left(\frac{|u|^{2}}{\left(\max \left\{L_{N},|u|\right\}\right)^{2}}\right) \\
& = \begin{cases}O\left(\frac{|u|^{2}}{L_{N}^{2}}\right), & \text { if }|u| \leq L_{N}, \\
O\left(\frac{|u|}{L_{N}}\right), & \text { if }|u|>L_{N} .\end{cases}
\end{aligned}
$$


Thus, from (5.25), we find that

$$
\begin{aligned}
\int_{-N}^{N} \frac{\cos (2 \pi k u)}{u^{2}} \int_{L_{N}}^{M_{N}}\{y\} h_{u}^{\prime}(y) d y d u= & O\left(\int_{-L_{N}}^{L_{N}} \frac{1}{u^{2}} \cdot \frac{u^{2}}{L_{N}^{2}} d u\right)+O\left(\int_{-N}^{-L_{N}} \frac{1}{u^{2}} \cdot \frac{|u|}{L_{N}} d u\right) \\
& +O\left(\int_{L_{N}}^{N} \frac{1}{u^{2}} \cdot \frac{|u|}{L_{N}} d u\right) \\
= & O\left(\frac{1}{L_{N}}\right)+O\left(\frac{\log N}{L_{N}}\right)=O\left(\frac{\log N}{L_{N}}\right) \cdot(5.26)
\end{aligned}
$$

Using (5.23), (5.24), and (5.26), we find that (5.19) now takes the form

$$
\begin{aligned}
\sum_{L_{N}<m \leq M_{N}} I_{N, m}= & -\frac{\pi x}{2 k}-\frac{1}{2 \pi k} \int_{-N}^{N} \int_{L_{N}}^{M_{N}} \frac{\cos (2 \pi k u)}{u^{2}} \sin ^{2}\left(\frac{\pi x u}{y}\right) d y d u \\
& +\frac{x}{2 k} \int_{-N}^{N} \frac{\cos (2 \pi k u)}{u} \sum_{L_{N}<m \leq M_{N}} \frac{1}{m} \sin \left(\frac{2 \pi x u}{m}\right) d u \\
& +O\left(\frac{N}{M_{N}}\right)+O\left(\frac{L_{N}}{N}\right)+O\left(\frac{\log N}{L_{N}}\right) .
\end{aligned}
$$

An examination of the error terms above shows that not only must $N / M_{N} \rightarrow 0$ as $N \rightarrow \infty$, but we also must require that $L_{N} / N \rightarrow 0$ and $\log N / L_{N} \rightarrow 0$ as $N \rightarrow \infty$.

Next, we apply the Euler-Maclaurin summation formula to estimate the remaining sum in (5.27). Set

$$
H_{u}(y):=\frac{1}{y} \sin \left(\frac{2 \pi x u}{y}\right)
$$

Then, by the Euler-Maclaurin formula,

$$
\sum_{L_{N}<m \leq M_{N}} \frac{1}{m} \sin \left(\frac{2 \pi x u}{m}\right)=\int_{L_{N}}^{M_{N}} \frac{1}{y} \sin \left(\frac{2 \pi x u}{y}\right) d y+\int_{L_{N}}^{M_{N}}\{y\} H_{u}^{\prime}(y) d y .
$$

Here,

$$
H_{u}^{\prime}(y)=-\frac{1}{y^{2}} \sin \left(\frac{2 \pi x u}{y}\right)-\frac{2 \pi x u}{y^{3}} \cos \left(\frac{2 \pi x u}{y}\right)=O\left(\frac{|u|}{y^{3}}\right)
$$

and hence

$$
\int_{L_{N}}^{M_{N}}\{y\} H_{u}^{\prime}(y) d y=O\left(\int_{L_{N}}^{M_{N}} \frac{|u|}{y^{3}} d y\right)=O\left(\frac{|u|}{L_{N}^{2}}\right) .
$$

This further implies that

$$
\int_{-N}^{N} \frac{\cos (2 \pi k u)}{u} \int_{L_{N}}^{M_{N}}\{y\} H_{u}^{\prime}(y) d y d u=O\left(\int_{-N}^{N} \frac{1}{|u|} \cdot \frac{|u|}{L_{N}^{2}} d u\right)=O\left(\frac{N}{L_{N}^{2}}\right) .
$$


Using (5.28) and (5.29) in (5.27), we find that

$$
\begin{aligned}
\sum_{L_{N}<m \leq M_{N}} I_{N, m}= & -\frac{\pi x}{2 k}-\frac{1}{2 \pi k} \int_{-N}^{N} \int_{L_{N}}^{M_{N}} \frac{\cos (2 \pi k u)}{u^{2}} \sin ^{2}\left(\frac{\pi x u}{y}\right) d y d u \\
& +\frac{x}{2 k} \int_{-N}^{N} \int_{L_{N}}^{M_{N}} \frac{\cos (2 \pi k u)}{u y} \sin \left(\frac{2 \pi x u}{y}\right) d y d u \\
& +O\left(\frac{N}{M_{N}}\right)+O\left(\frac{L_{N}}{N}\right)+O\left(\frac{\log N}{L_{N}}\right)+O\left(\frac{N}{L_{N}^{2}}\right) .
\end{aligned}
$$

Examining the error term in (5.30), we see that $L_{N}$ needs to be chosen so that both $\sqrt{N} / L_{N} \rightarrow 0$ and $L_{N} / N \rightarrow 0$, as $N \rightarrow \infty$. As mentioned before, we also need to choose $M_{N}$ such that $N / M_{N} \rightarrow 0$ as $N \rightarrow \infty$.

Next, in the second double integral on the right side of (5.30), we integrate by parts with respect to $y$ to obtain

$$
\begin{aligned}
& \frac{x}{2 k} \int_{-N}^{N} \int_{L_{N}}^{M_{N}} \frac{\cos (2 \pi k u)}{u y} \sin \left(\frac{2 \pi x u}{y}\right) d y d u \\
&=\frac{1}{4 \pi k} \int_{-N}^{N} \int_{L_{N}}^{M_{N}} \frac{\cos (2 \pi k u)}{u^{2}} y \frac{\partial}{\partial y} \cos \left(\frac{2 \pi x u}{y}\right) d y d u \\
&=\frac{1}{4 \pi k} \int_{-N}^{N} \frac{\cos (2 \pi k u)}{u^{2}}\left(M_{N} \cos \left(\frac{2 \pi x u}{M_{N}}\right)\right. \\
&\left.-L_{N} \cos \left(\frac{2 \pi x u}{L_{N}}\right)-\int_{L_{N}}^{M_{N}} \cos \left(\frac{2 \pi x u}{y}\right) d y\right) d u .
\end{aligned}
$$

Using the identity $\cos (2 a)=1-2 \sin ^{2} a$ for each of the three cosines appearing on the right side of (5.31), we find that the three terms $M_{N}-L_{N}-\int_{L_{N}}^{M_{N}} d y$ cancel. We can therefore write (5.31) in the form

$$
\begin{aligned}
\frac{x}{2 k} \int_{-N}^{N} \int_{L_{N}}^{M_{N}} \frac{\cos (2 \pi k u)}{u y} \sin \left(\frac{2 \pi x u}{y}\right) d y d u \\
=\frac{1}{2 \pi k} \int_{-N}^{N} \frac{\cos (2 \pi k u)}{u^{2}}\left(-M_{N} \sin ^{2}\left(\frac{\pi x u}{M_{N}}\right)\right. \\
\left.\quad+L_{N} \sin ^{2}\left(\frac{\pi x u}{L_{N}}\right)+\int_{L_{N}}^{M_{N}} \sin ^{2}\left(\frac{\pi x u}{y}\right) d y\right) d u \\
=-\frac{M_{N}}{2 \pi k} \int_{-N}^{N} \frac{\cos (2 \pi k u)}{u^{2}} \sin ^{2}\left(\frac{\pi x u}{M_{N}}\right) d u+\frac{L_{N}}{2 \pi k} \int_{-N}^{N} \frac{\cos (2 \pi k u)}{u^{2}} \sin ^{2}\left(\frac{\pi x u}{L_{N}}\right) d u \\
\quad+\frac{1}{2 \pi k} \int_{-N}^{N} \int_{L_{N}}^{M_{N}} \frac{\cos (2 \pi k u)}{u^{2}} \sin ^{2}\left(\frac{\pi x u}{y}\right) d y d u .
\end{aligned}
$$


Putting (5.32) in (5.30), we see that the first double integral on the right side of (5.30) cancels. Hence,

$$
\begin{aligned}
\sum_{L_{N}<m \leq M_{N}} I_{N, m}= & -\frac{\pi x}{2 k}-\frac{M_{N}}{2 \pi k} \int_{-N}^{N} \frac{\cos (2 \pi k u)}{u^{2}} \sin ^{2}\left(\frac{\pi x u}{M_{N}}\right) d u \\
& +\frac{L_{N}}{2 \pi k} \int_{-N}^{N} \frac{\cos (2 \pi k u)}{u^{2}} \sin ^{2}\left(\frac{\pi x u}{L_{N}}\right) d u \\
& +O\left(\frac{N}{M_{N}}\right)+O\left(\frac{L_{N}}{N}\right)+O\left(\frac{\log N}{L_{N}}\right)+O\left(\frac{N}{L_{N}^{2}}\right) .
\end{aligned}
$$

In the two integrals in (5.33), we make the changes of variable $z=\pi x u / M_{N}$ and $z=\pi x u / L_{N}$, respectively. First,

$$
\begin{aligned}
-\frac{M_{N}}{2 \pi k} \int_{-N}^{N} \frac{\cos (2 \pi k u)}{u^{2}} \sin ^{2}\left(\frac{\pi x u}{M_{N}}\right) d u & =-\frac{x}{2 k} \int_{-\pi x N / M_{N}}^{\pi x N / M_{N}} \frac{\cos \left(2 k M_{N} z / x\right)}{z^{2}} \sin ^{2} z d z \\
& =O\left(\frac{N}{M_{N}}\right) .
\end{aligned}
$$

Secondly,

$$
\frac{L_{N}}{2 \pi k} \int_{-N}^{N} \frac{\cos (2 \pi k u)}{u^{2}} \sin ^{2}\left(\frac{\pi x u}{L_{N}}\right) d u=\frac{x}{2 k} \int_{-\pi x N / L_{N}}^{\pi x N / L_{N}} \frac{\cos \left(2 k L_{N} z / x\right)}{z^{2}} \sin ^{2} z d z
$$

The integral on the right side of (5.35) is over an interval that is much larger than that on the right side of (5.34), since, as we have previously prescribed, we must require that $N / L_{N}$ approach $\infty$ as $N \rightarrow \infty$. In order to estimate this integral, we need to take advantage of the highly oscillatory behavior of the factor $\cos \left(2 k L_{N} z / x\right)$. Integrating by parts, we therefore deduce that

$$
\begin{gathered}
\frac{x}{2 k} \int_{-\pi x N / L_{N}}^{\pi x N / L_{N}} \frac{\cos \left(2 k L_{N} z / x\right)}{z^{2}} \sin ^{2} z d z=-\frac{x^{2}}{4 k^{2} L_{N}} \int_{-\pi x N / L_{N}}^{\pi x N / L_{N}} \sin \left(2 k L_{N} z / x\right) \frac{d}{d z} \frac{\sin ^{2} z}{z^{2}} d z \\
=-\frac{x^{2}}{4 k^{2} L_{N}} \int_{-\pi x N / L_{N}}^{\pi x N / L_{N}} \sin \left(2 k L_{N} z / x\right) \frac{z \sin (2 z)-2 \sin ^{2} z}{z^{3}} d z \\
=O\left(\frac{1}{L_{N}} \int_{-\infty}^{\infty}\left|\frac{z \sin (2 z)-2 \sin ^{2} z}{z^{3}}\right| d z\right)=O\left(\frac{1}{L_{N}}\right)
\end{gathered}
$$

since $\left(z \sin (2 z)-2 \sin ^{2} z\right) / z^{3}$ is analytic at the origin and $O\left(1 / z^{2}\right)$ as $z \rightarrow \pm \infty$. Hence, using (5.34) and (5.36) in (5.33), we conclude that

$$
\sum_{L_{N}<m \leq M_{N}} I_{N, m}=-\frac{\pi x}{2 k}+O\left(\frac{N}{M_{N}}\right)+O\left(\frac{L_{N}}{N}\right)+O\left(\frac{\log N}{L_{N}}\right)+O\left(\frac{N}{L_{N}^{2}}\right) .
$$

Therefore, subject to the conditions

$$
\frac{N}{M_{N}} \rightarrow 0, \quad \frac{L_{N}}{N} \rightarrow 0, \quad \frac{\sqrt{N}}{L_{N}} \rightarrow 0, \quad \text { as } N \rightarrow \infty,
$$


we can deduce that

$$
\lim _{N \rightarrow \infty} \sum_{L_{N}<m \leq M_{N}} I_{N, m}=-\frac{\pi x}{2 k} .
$$

We can easily choose $M_{N}$ so that the first condition in (5.38) is satisfied. Naturally, we prefer that (5.39) holds under the largest possible range of $m$, and so we want to choose $L_{N}$ as small as possible. Therefore, we choose $L_{N}$ slightly larger than $\sqrt{N}$, so that $\sqrt{N} / L_{N} \rightarrow 0$ as $N \rightarrow \infty$. Subject to this condition, by (5.39) and (5.38), we deduce that (5.18) will be established if we can show that

$$
\lim _{N \rightarrow \infty} \sum_{1 \leq m \leq L_{N}} I_{N, m}=\frac{\pi}{2} F\left(\frac{x}{k}\right) .
$$

Choose now a large $\mathrm{N}$, and choose $L_{N}$ subject to the growth condition introduced above. Choose $m$ such that $1 \leq m \leq L_{N}$. By (5.12),

$$
\begin{aligned}
I_{N, m}= & \int_{-N}^{N} \frac{\sin (2 \pi k u)}{u} \sin ^{2}\left(\frac{\pi x u}{m}\right) d u \\
= & \frac{1}{2} \int_{-N}^{N} \frac{\sin (2 \pi k u)}{u}\left(1-\cos \left(\frac{2 \pi x u}{m}\right)\right) d u \\
= & \frac{1}{2} \int_{-N}^{N} \frac{\sin (2 \pi k u)}{u} d u-\frac{1}{4} \int_{-N}^{N} \frac{\sin \left(2 \pi k u+\frac{2 \pi x u}{m}\right)}{u} d u \\
& -\frac{1}{4} \int_{-N}^{N} \frac{\sin \left(2 \pi k u-\frac{2 \pi x u}{m}\right)}{u} d u
\end{aligned}
$$

We first examine the first integral on the far right side of (5.41). Letting $z=2 \pi k u$, we find that

$$
\frac{1}{2} \int_{-N}^{N} \frac{\sin (2 \pi k u)}{u} d u=\frac{1}{2} \int_{-2 \pi k N}^{2 \pi k N} \frac{\sin z}{z} d z=\frac{\pi}{2}+O\left(\frac{1}{N}\right) .
$$

Similarly, the second integral on the right side of (5.41) equals

$$
\begin{aligned}
-\frac{1}{4} \int_{-N}^{N} \frac{\sin \left(2 \pi k u+\frac{2 \pi x u}{m}\right)}{u} d u & =-\frac{1}{4} \int_{-\left(2 \pi k+\frac{2 \pi x}{m}\right) N}^{\left(2 \pi k+\frac{2 \pi x}{m}\right) N} \frac{\sin z}{z} d z \\
& =-\frac{\pi}{4}+O\left(\frac{1}{\left(2 \pi k+\frac{2 \pi x}{m}\right) N}\right)=-\frac{\pi}{4}+O\left(\frac{1}{N}\right)
\end{aligned}
$$

uniformly for $m$ in the given range, $1 \leq m \leq L_{N}$. In examining the third integral on the far right side of (5.41), we need to take into consideration the possibility that the quantity $2 \pi k-2 \pi x / m$ is small, or possibly even 0 . This last possibility can happen only if $k=x / m$, which can only happen if $x / k$ is an integer. If such is the case, then there is a unique $m$, which will be in the given range $1 \leq m \leq L_{N}$ if $L_{N}$ is sufficiently large. In this situation, we obviously have

$$
-\frac{1}{4} \int_{-N}^{N} \frac{\sin \left(2 \pi k u-\frac{2 \pi x u}{m}\right)}{u} d u=0 .
$$


Next, for $m$ large enough in terms of $k$ and $x$, the quantity $2 \pi k-2 \pi x / m$ stays away from the origin, and so we may proceed as above. To be precise, for $m \geq 2 x / k$, we have $2 \pi k-2 \pi x / m \geq \pi k$, and hence

$$
\begin{aligned}
-\frac{1}{4} \int_{-N}^{N} \frac{\sin \left(2 \pi k u-\frac{2 \pi x u}{m}\right)}{u} d u & =-\frac{1}{4} \int_{-\left(2 \pi k-\frac{2 \pi x}{m}\right) N}^{\left(2 \pi k-\frac{2 \pi x}{m}\right) N} \frac{\sin z}{z} d z \\
& =-\frac{\pi}{4}+O\left(\frac{1}{\left(2 \pi k-\frac{2 \pi x}{m}\right) N}\right) \\
& =-\frac{\pi}{4}+O\left(\frac{1}{\pi k N}\right)=-\frac{\pi}{4}+O\left(\frac{1}{N}\right) .
\end{aligned}
$$

Now let $1 \leq m \leq 2 x / k$ and suppose that $m$ is not equal to $x / k$ in the case that $x / k$ is an integer. Then in this finite range, the quantity $|2 \pi k-2 \pi x / m|$ has a minimum which is strictly positive and depends on $x$ and $k$ only. Denote this minimum by $\delta(x, k)$ and distinguish two cases.

First, if $x / k<m<2 x / k$, then $2 \pi k-2 \pi x / m \geq \delta(x, k)$, and, as in (5.45), we find that

$$
-\frac{1}{4} \int_{-N}^{N} \frac{\sin \left(2 \pi k u-\frac{2 \pi x u}{m}\right)}{u} d u=-\frac{\pi}{4}+O\left(\frac{1}{\delta(x, k) N}\right)=-\frac{\pi}{4}+O\left(\frac{1}{N}\right) .
$$

Secondly, if $1 \leq m<x / k$, then $2 \pi k-2 \pi x / m \leq-\delta(x, k)$, and

$$
\begin{aligned}
-\frac{1}{4} \int_{-N}^{N} \frac{\sin \left(2 \pi k u-\frac{2 \pi x u}{m}\right)}{u} d u & =-\frac{1}{4} \int_{-\left(2 \pi k-\frac{2 \pi x}{m}\right) N}^{\left(2 \pi k-\frac{2 \pi x}{m}\right) N} \frac{\sin z}{z} d z \\
& =\frac{1}{4} \int_{-\left|2 \pi k-\frac{2 \pi x}{m}\right| N}^{\left|2 \pi k-\frac{2 \pi x}{m}\right| N} \frac{\sin z}{z} d z \\
& =\frac{\pi}{4}+O\left(\frac{1}{\delta(x, k) N}\right)=\frac{\pi}{4}+O\left(\frac{1}{N}\right) .
\end{aligned}
$$

Combining (5.41) and (5.42)-(5.47), we conclude that

$$
I_{N, m}= \begin{cases}\frac{\pi}{2}+O\left(\frac{1}{N}\right), & \text { if } m<\frac{x}{k}, \\ \frac{\pi}{4}+O\left(\frac{1}{N}\right), & \text { if } m=\frac{x}{k} \\ O\left(\frac{1}{N}\right), & \text { if } m>\frac{x}{k} .\end{cases}
$$

Adding the relations (5.48) for $1 \leq m \leq L_{N}$, we finally deduce that

$$
\sum_{1 \leq m \leq L_{N}} I_{N, m}= \begin{cases}{\left[\frac{x}{k}\right] \frac{\pi}{2}+O\left(\frac{L_{N}}{N}\right),} & \text { if } \frac{x}{k} \text { is not an integer } \\ \left(\frac{x}{k}-\frac{1}{2}\right) \frac{\pi}{2}+O\left(\frac{L_{N}}{N}\right), & \text { if } \frac{x}{k} \text { is an integer. }\end{cases}
$$


Recall that $L_{N}$ was chosen to be slightly larger than $\sqrt{N}$, so that the error terms on the right-hand side of (5.49) tend to 0 as $N$ tends to $\infty$. We therefore conclude that

$$
\lim _{N \rightarrow \infty} \sum_{1 \leq m \leq L_{N}} I_{N, m}= \begin{cases}{\left[\frac{x}{k}\right] \frac{\pi}{2},} & \text { if } \frac{x}{k} \text { is not an integer }, \\ \left(\frac{x}{k}-\frac{1}{2}\right) \frac{\pi}{2}, & \text { if } \frac{x}{k} \text { is an integer. }\end{cases}
$$

Taking into account the definition of $F(x)$ in (1.2), we see that (5.50) establishes the desired equality (5.40), and hence it also establishes Theorem 3.1, for all $x$ and $\theta$ in the given ranges $x>0$ and $0<\theta<1$.

The authors are grateful to O-Yeat Chan for numerical calculations of both interpretations of Ramanujan's Bessel series identity.

\section{REFERENCES}

[1] B. C. Berndt, Identities involving the coefficients of a class of Dirichlet series, I, Trans. Amer. Math. Soc. 137 (1969), 345-359.

[2] B. C. Berndt, Identities involving the coefficients of a class of Dirichlet series, VII, Trans. Amer. Math. Soc. 201 (1975), 247-261.

[3] B. C. Berndt, Periodic Bernoulli numbers, summation formulas and applications in Theory and Application of Special Functions, R. A. Askey, ed., Academic Press, New York, 1975.

[4] B. C. Berndt, Classical theorems on quadratic residues, L'Enseign. Math. 22 (1976), 261-304.

[5] B. C. Berndt, R. J. Evans, and K. S. Williams, Gauss and Jacobi Sums, John Wiley, New York, 1998.

[6] K. Chandrasekharan and R. Narasimhan, Hecke's functional equation and arithmetical identities, Ann. of Math. 74 (1961), 1-23.

[7] K. Chandrasekharan and R. Narasimhan, Functional equations with multiple gamma factors and the average order of arithmetical functions, Ann. of Math. 76 (1962), 93-136.

[8] I. S. Gradshteyn and I. M. Ryzhik, eds., Table of Integrals, Series, and Products, 5th ed., Academic Press, San Diego, 1994.

[9] G. H. Hardy, On the expression of a number as the sum of two squares, Quart. J. Math. (Oxford) 46 (1915), 263-283.

[10] G. H. Hardy, Collected Papers, Vol. II, Oxford University Press, Oxford, 1967.

[11] N. M. Huxley, Exponential sums and lattice points. III, Proc. London Math. Soc. (3) 87 (2003), 591-609.

[12] C. G. J. Jacobi, Fundamenta Nova Theoriae Functionum Ellipticarum, Sumptibus, Fratrum Bornträger, Regiomonti, 1829.

[13] T. Kano, On the Bessel-series expression for $\sum \frac{1}{n} \sin \frac{x}{n}$, Math. J. Okayama Univ. 16 (1974), $129-136$.

[14] S. Ramanujan, The Lost Notebook and Other Unpublished Papers, Narosa, New Delhi, 1988.

[15] S. L. Segal, On $\sum 1 / n \sin (x / n)$, J. London Math. Soc. (2) 4 (1972), 385-393.

[16] E. C. Titchmarsh, Theory of Fourier Integrals, 2nd ed., Clarendon Press, Oxford, 1948.

[17] G. N. Watson, A Treatise on the Theory of Bessel Functions, 2nd ed., University Press, Cambridge, 1966 .

Department of Mathematics, University of Illinois, 1409 West Green Street, UrBANA, IL 61801, USA

E-mail address: berndt@math.uiuc.edu

Department of Mathematics, University of Illinois, 1409 West Green Street, UrBANA, IL 61801, USA 
Institute of Mathematics of the Romanian Academy, P.O. Box 1-764, Bucharest RO-70700, ROMANiA

E-mail address: zaharesc@math.uiuc.edu 